\title{
Identification of a transcriptome profile associated with improvement of organ function in septic shock patients after early supportive therapy
}

Matteo Barcella ${ }^{1,2+}$, Bernardo Bollen Pinto ${ }^{3 \dagger}$, Daniele Braga ${ }^{1,2}$, Francesca D'Avila ${ }^{1,2}$, Federico Tagliaferri ${ }^{1,2}$, Marie-Angelique Cazalis ${ }^{4}$, Guillaume Monneret ${ }^{5}$, Antoine Herpain ${ }^{6}$, Karim Bendjelid ${ }^{3+}$ and Cristina Barlassina ${ }^{1,2^{*+}}$ (D)

\begin{abstract}
Background: Septic shock is the most severe complication of sepsis and this syndrome is associated with high mortality. Treatment of septic shock remains largely supportive of hemodynamics and tissue perfusion. Early changes in organ function assessed by the Sequential Organ Function Assessment (SOFA) score are highly predictive of the outcome. However, the individual patient's response to supportive therapy is very heterogeneous, and the mechanisms underlying this variable response remain elusive. The aim of the study was to investigate the transcriptome of whole blood in septic shock patients with different responses to early supportive hemodynamic therapy assessed by changes in SOFA scores within the first $48 \mathrm{~h}$ from intensive care unit (ICU) admission.
\end{abstract}

Methods: We performed whole blood RNA sequencing in 31 patients: 17 classified as responders (R) and 14 as nonresponders (NR). Gene expression was investigated at ICU admission (time point 1, or T1), comparing R with NR [padj $<0.01$; Benjamini-Hochberg (BH)] and over time from T1 to T2 (48 h later) in R and NR independently (paired analysis, padj $<0.01 ; \mathrm{BH}$ ). Then the differences in gene expression trends over time were evaluated (Mann-Whitney, $P<0.01$ ). To identify enriched biological processes, we performed an over-representation analysis based on a right-sided hypergeometric test with Bonferroni step-down as multiple testing correction (padj < 0.05).

Results: At ICU admission, we did not identify differentially expressed genes (DEGs) between the two groups. In the transition from T1 to T2, the activation of genes involved in T cell-mediated immunity, granulocyte and natural killer (NK) cell functions, and pathogen lipid clearance was noted in the R group. Genes involved in acute inflammation were downregulated in both groups.

Conclusions: Within the limits of a small sample size, our results could suggest that early activation of genes of the adaptive immune response is associated with an improvement in organ function.

Keywords: Critical illness, SOFA score, Gene expression, RNA-Seq, Septic shock

\footnotetext{
* Correspondence: cristina.barlassina@unimi.it

${ }^{+}$Matteo Barcella, Bernardo Bollen Pinto, Karim Bendjelid and Cristina

Barlassina contributed equally to this work.

${ }^{1}$ Dipartimento di Scienze della Salute, Università degli Studi di Milano, Via

Rudini 8, 20142 Milan, Italy

${ }^{2}$ Fondazione Filarete, Viale Ortles 22/4, 20139 Milan, Italy

Full list of author information is available at the end of the article
}

(c) The Author(s). 2018 Open Access This article is distributed under the terms of the Creative Commons Attribution 4.0 International License (http://creativecommons.org/licenses/by/4.0/), which permits unrestricted use, distribution, and reproduction in any medium, provided you give appropriate credit to the original author(s) and the source, provide a link to the Creative Commons license, and indicate if changes were made. The Creative Commons Public Domain Dedication waiver (http://creativecommons.org/publicdomain/zero/1.0/) applies to the data made available in this article, unless otherwise stated. 


\section{Introduction}

Sepsis is a life-threatening organ dysfunction caused by a dysregulated immune response to infection [1], and septic shock is its most severe form and is associated with an increased risk of mortality [2]. In addition to antibiotics or source control using surgery (or both), treatment of septic shock remains largely supportive of hemodynamics and tissue perfusion with fluids and vasopressors, which are associated with harmful side effects and can have a direct impact on immunity, organ function, and mortality [3, 4]. Early changes in organ function during the first days of an intensive care unit (ICU) stay as assessed by the Sequential Organ Function Assessment (SOFA) score are highly predictive of final outcome [5]. However, the response to supportive therapy is highly heterogeneous across patients, and the mechanisms underlying such variability remain elusive. In this context, it is not surprising that applying a "onesize fits all" protocol of fluids and vasopressors in early septic shock did not result in an amelioration of the outcome in all patients [6].

The characterization of patients at the molecular level is a promising approach to identify more specific targets for new therapeutic interventions in selected groups of patients [7]. Transcriptome profiling is a powerful tool to investigate and characterize the molecular mechanisms underlying specific physiological or pathological conditions.

In patients with septic shock, major transcriptomic changes occur within the first $48 \mathrm{~h}$ of shock development [8] and are associated with mortality in the ICU [9]. However, the reasons for this association remain largely unexplored. The authors hypothesized that this could be due to different trajectories of organ (dys)function in response to standard therapy.

We performed a whole blood RNA sequencing (RNASeq) analysis in septic shock patients with different responses to early supportive hemodynamic therapy as assessed by changes in SOFA scores within the first $48 \mathrm{~h}$ from ICU admission. The aim of the study was to identify gene expression profiles that distinguish patients as early improving (responders) or not (non-responders) on the basis of their SOFA score following initial therapy. Some of the results of this study have been previously reported in the form of an abstract [10].

\section{Methods}

A detailed description of the methods is provided in Additional file 1 .

\section{Study design and participants}

This study is part of the multicenter prospective observational trial ShockOmics (ClinicalTrials.gov Identifier: NCT02141607) [11]. The ICUs involved are located in the Hopitaux Universitaires de Genève, Universite de Geneve (Geneva, Switzerland) and Hospital Erasme, Universite Libre de Bruxelles (Brussels, Belgium). The ethics committees of the two participating institutions approved the clinical protocol. Informed consent was obtained from the patients or their representatives. As previously described [11], we included consecutive adult ( $>18$ years old) patients admitted for septic shock in the ICUs of two university tertiary hospital centers with an admission SOFA score at least 6 and an arterial lactate at least $2 \mathrm{mmol} / \mathrm{L}$. Septic shock was defined in accordance with the current recommendations and international guidelines at that time [12]. Exclusion criteria included expected death within $24 \mathrm{~h}$ of ICU admission; more than 4 units of red blood cells or more than 1 fresh frozen plasma transfused; active hematological malignancy; metastatic cancer; chronic immunosuppression; pre-existing end-stage renal disease requiring renal replacement therapy; recent cardiac surgery; Child-Pugh $\mathrm{C}$ cirrhosis; and terminal illness.

We used SOFA score changes between time point 1 (T1), which was within $16 \mathrm{~h}$ of ICU admission, and time point 2 (T2), which was $48 \mathrm{~h}$ after study enrolment as an index of response to early supportive treatment to stratify subjects as responders (Rs) or non-responders (NRs) as previously reported [13]. A subject was classified as NR if the difference in SOFA score between T1 and T2 was less than 5 and the SOFA score at T2 was greater than 8 . Patients who did not meet these criteria were classified as $\mathrm{R}$.

\section{Blood collection and RNA extraction}

Peripheral blood was collected in EDTA tubes at T1 and $\mathrm{T} 2$ and stored at $-20{ }^{\circ} \mathrm{C}$ after adding $400 \mu \mathrm{L}$ of $2 \mathrm{X}$ Denaturing solution (Ambion, Austin, TX, USA). Total RNA was extracted from $800 \mu \mathrm{L}$ of blood using the MirVana Paris Kit and treated with Turbo DNA-free Kit (Ambion). RNA quality was assessed on an Agilent Bioanalyzer using the RNA 6000 Nano Kit (Agilent, Santa Clara, CA, USA), and samples were considered suitable for processing if the RNA integrity number was greater than 7.5 .

\section{Library preparation}

Sequencing libraries were prepared using TruSeq Stranded Total RNA obtained from the Ribo-Zero Globin Kit (Illumina, San Diego, CA, USA) using $800 \mathrm{ng}$ of total RNA. Final libraries were validated with the Agilent DNA1000 kit and sequenced on a HiSeq2500 platform, producing $50 \times 2$-base pair paired-end reads.

\section{Sequencing data analysis}

High-quality paired-end reads were aligned to the human reference genome (GRCh38) using STAR (version 2.5.2b) [14], and only uniquely mapping reads were used. 
Reads were assigned to genes with featureCounts (version 1.5.1) [15] using the gencode (version 25) primary assembly gene transfer file (GTF) as a reference annotation file for genomic feature boundaries.

\section{Differential expression analysis}

Data preprocessing, exploratory data analysis, and analysis of differential gene expression were performed using DESeq2 package [16] built-in functions. We studied gene expression differences between $\mathrm{R}$ and NR at T1 and gene expression changes over time. These data were compared between T2 to T1 in R and NR groups, separately, using paired analysis. For both analyses, genes with padj $<0.01-$ Benjamini-Hochberg multiple test correction (FDR)-were considered differentially expressed (DEGs).

After selecting all the genes identified as DEGs in R or NR over time, we applied a Mann-Whitney test to compare T2 versus T1 fold changes between $R$ and NR groups to identify DEGs with significant different trends between $\mathrm{R}$ and NR. The significance threshold was set to a $P$ value of less than 0.01 .

\section{Biological processes analysis}

We used ClueGO version 2.3.4 [17] to identify enriched biological processes starting from the lists of DEGs identified in the over-time analysis in R and NR and the DEGs common to the two groups. The lists were used as input for the analysis after filtering for padj $<0.001$. We performed an over-representation analysis (ORA) based on a right-sided hypergeometric test that uses Bonferroni step down as multiple testing correction. Enriched terms were grouped using kappa statistics, and only enriched clusters with a $P$ value of less than 0.05 were considered statistically significant.

\section{Validation in an external dataset}

To validate the DEGs identified in the transition from ICU admission to $48 \mathrm{~h}$ later, we searched in Gene Expression Omnibus (GEO) and Array Express repositories for gene expression datasets of septic shock patients studied at multiple time points. The microarray expression dataset GSE57065 included 28 septic patients studied at the onset of shock (that is, within $30 \mathrm{~min}$ after the beginning of vasoactive treatment, $\mathrm{H} 0$ ) and 24 and $48 \mathrm{~h}$ after (H24 and H48) [8]. We stratified patients as Rs or NRs using SOFA score changes between $\mathrm{HO}$ and $\mathrm{H} 48$ as described in the "Study design and participants" section. Patients missing the $\mathrm{H} 48$ blood sample were excluded from the validation that was performed on 17 Rs and 9 NRs. We compared gene expression between $\mathrm{HO}$ and $\mathrm{H} 48$ in the $\mathrm{R}$ and NR groups, separately. We established the analysis modifying the GEO2R script to take into account paired data [18]. Genes with padj < 0.01-Benjamini-Hochberg multiple test correction (FDR) - and $\log 2 \mathrm{FC}>|0.5|$ were considered DEGs.

\section{Results \\ Clinical variables}

The study included 31 patients with septic shock enrolled between November 2014 and March 2016. In total, 17 patients were classified as $\mathrm{R}$ and 14 were classified as NR. The clinical characteristics of Rs and NRs at $\mathrm{T} 1$ and $\mathrm{T} 2$ are summarized in Table 1.

At T1, no significant differences were noted between the two groups in terms of variables describing the severity of organ dysfunction, whereas, consistent with our definition of $\mathrm{R}$ and NR, the SOFA score at T2 was increased in NRs $(P<0.001)$. No significant differences were noted between the two groups regarding the source of infection, circulating markers of inflammation, or leukocyte and lymphocyte counts. The length of the ICU stay was longer in NR patients $(P<0.05)$. More patients died in the ICU in the NR group (4 out of 14 in NRs versus 2 out of 17 in Rs); however, this difference was not statistically significant.

\section{Sequencing experiment}

Total RNA libraries were sequenced in several batches, producing $31.07 \mathrm{M} \pm 6.92 \mathrm{M}$ and $29.33 \mathrm{M} \pm 7.26 \mathrm{M}$ raw read pairs on average for Rs and NRs, respectively. Ribosomal depletion was effective for all samples; the rRNA rate on mapped data was negligible in both groups $(0.80$ $\pm 0.95 \%$ and $0.78 \pm 0.82 \%$ for Rs and NRs, respectively). The percentages of reads mapping to exons (86.09 \pm $5.83 \%$ exonic rate) and DNase efficiency $(2.75 \pm 2.02 \%$ intergenic rate) were satisfactory in all samples. We obtained on average $13.67 \pm 3.77$ and $13.41 \pm 3.69$ million of uniquely and unambiguously mapped fragments for Rs and NRs, respectively (Additional file 2: Table S1). Globin reduction was successful with average percentages of counts mapping on globin genes of approximately $0.13 \%$ and $0.14 \%$ for NRs and Rs, respectively. Moreover, depletion efficiency was not statistically different for any of the genes coding for globins (Additional file 3: Table S7). Although we cannot test whether globin reduction influences sequencing efficiency of non-globin genes, this would equally affect both NR and R samples and thus is likely to bias toward the null.

\section{Gene expression analysis at ICU admission}

We explored whether the transcriptome profile could distinguish Rs from NRs at ICU admission (T1). Principal component analysis was performed on the 2000 most variable genes across samples and did not reveal any separation between the R and NR groups (Fig. 1). Indeed, we could not identify any statistically significant DEG at T1 between Rs and NRs (padj < 0.01). 
Table 1 Clinical variables

\begin{tabular}{|c|c|c|c|}
\hline & Responders $(n=17)$ & Non-responders $(n=14)$ & $P$ value \\
\hline \multicolumn{4}{|l|}{ Demographic parameters } \\
\hline Age, years & $62.8(18.4)$ & $68.6(20.7)$ & 0.42 \\
\hline Sex, female & $5(29 \%)$ & $5(36 \%)$ & 0.71 \\
\hline Body mass index, $\mathrm{kg} / \mathrm{m}^{2}$ & $27.0(4.6)$ & $26.9(6.4)$ & 0.98 \\
\hline \multicolumn{4}{|l|}{ Source of infection } \\
\hline Abdominal & $5(29 \%)$ & $5(36 \%)$ & \multirow[t]{4}{*}{0.22} \\
\hline Respiratory & $4(24 \%)$ & $7(50 \%)$ & \\
\hline Urinary tract & $6(35 \%)$ & $1(7 \%)$ & \\
\hline Other & $2(12 \%)$ & $1(7 \%)$ & \\
\hline \multicolumn{4}{|l|}{ Clinical parameters } \\
\hline Length of stay in ICU, days & $6.2(4.9)$ & $11.9(6.3)$ & 0.01 \\
\hline Length of stay in hospital, days & $21.0(18.6)$ & $28.7(17.9)$ & 0.31 \\
\hline Death in ICU & $2(12 \%)$ & $4(29 \%)$ & 0.24 \\
\hline Death in hospital & $2(12 \%)$ & $5(36 \%)$ & 0.11 \\
\hline APACHE $\|$ & $23.1(6.8)$ & $25.1(7.2)$ & 0.42 \\
\hline SOFA T1 & $11.6(3.0)$ & $12.4(2.4)$ & 0.39 \\
\hline SOFA T2 & $6.1(1.6)$ & $11.4(2.6)$ & $P<0.001$ \\
\hline Temperature, ${ }^{\circ} \mathrm{C}, \mathrm{T} 1$ & $37.2(1.2)$ & $37.8(1.2)$ & 0.17 \\
\hline Temperature, ${ }^{\circ} \mathrm{C}, \mathrm{T} 2$ & $37.8(0.8)$ & $37.2(0.8)$ & 0.02 \\
\hline Lactate, mmol/L, T1 & $4.0(1.8)$ & $5.3(2.8)$ & 0.17 \\
\hline Lactate, $\mathrm{mmol} / \mathrm{L}, \mathrm{T} 2$ & $1.5(0.8)$ & $2.1(0.9)$ & 0.10 \\
\hline $\mathrm{SvCO}_{2}, \%, \mathrm{~T} 1$ & $75.0(5.57)$ & $74.36(7.72)$ & 0.82 \\
\hline $\mathrm{SvCO}_{2}, \%, \mathrm{~T} 2$ & $68.5(6.84)$ & $69.33(9.38)$ & 0.84 \\
\hline Heart rate, bpm, $\mathrm{T} 1$ & $105.8(28.8)$ & $109.2(17.7)$ & 0.69 \\
\hline Heart rate, bpm, T2 & $89.2(23.2)$ & $95.4(21.8)$ & 0.46 \\
\hline Mean arterial pressure, mm Hg, $\mathrm{T1}$ & $60.1(4.7)$ & $57.4(7.3)$ & 0.24 \\
\hline Mean arterial pressure, mm Hg, $\mathrm{T} 2$ & $67.5(9.9)$ & $66.0(7.0)$ & 0.62 \\
\hline Hydrocortisone T1 & $0(0 \%)$ & $0(0 \%)$ & - \\
\hline Hydrocortisone T2 & $1(6 \%)$ & $0(0 \%)$ & 0.36 \\
\hline Vasopressor treatment T1 & $17(100 \%)$ & $14(100 \%)$ & 0.36 \\
\hline Vasopressor treatment T2 & $3(18 \%)$ & $14(100 \%)$ & $P<0.001$ \\
\hline Norepinehrine dose, $\mu \mathrm{g} / \mathrm{kg}$ per min, $\mathrm{T} 1$ & $0.26(0.19)$ & $0.49(0.38)$ & 0.06 \\
\hline Norepinehrine dose, $\mu \mathrm{g} / \mathrm{kg}$ per min, T2 & $0.09(0.04)$ & $0.24(0.33)$ & 0.12 \\
\hline Fluid balance, mL, T1 & $2229(2468)$ & 3498 (1943) & 0.12 \\
\hline Fluid balance, mL, $\mathrm{T2}$ & 135 (1203) & $1566(2062)$ & 0.03 \\
\hline Urinary output, mL, T1 & $1674(745)$ & $1194(750)$ & 0.09 \\
\hline Urinary output, mL, T2 & $2273(849)$ & $2040(1691)$ & 0.65 \\
\hline Creatinine, mg/dL, T1 & $1.73(1.17)$ & $1.65(0.83)$ & 0.83 \\
\hline Creatinine, mg/dL, T2 & $1.20(1.01)$ & $1.46(0.95)$ & 0.46 \\
\hline Renal replacement therapy $\mathrm{T1}$ & $1(6 \%)$ & $0(0 \%)$ & 0.36 \\
\hline Renal replacement therapy T2 & $0(0 \%)$ & $0(0 \%)$ & - \\
\hline Mechanical ventilation $\mathrm{T1}$ & $13(76 \%)$ & $13(93 \%)$ & 0.22 \\
\hline Mechanical ventilation T2 & $9(53 \%)$ & $11(79 \%)$ & 0.14 \\
\hline $\mathrm{paO}_{2} / \mathrm{FiO}_{2}, \mathrm{~mm} \mathrm{Hg}, \mathrm{T} 1$ & $203.9(110.5)$ & $205.7(147.4)$ & 0.97 \\
\hline
\end{tabular}


Table 1 Clinical variables (Continued)

\begin{tabular}{llll}
\hline & Responders $(n=17)$ & Non-responders $(n=14)$ & $P$ value \\
\hline $\mathrm{paO}_{2} / \mathrm{FiO}_{2}, \mathrm{~mm} \mathrm{Hg}, \mathrm{T} 2$ & $271.0(102.3)$ & $219.4(48.6)$ & 0.08 \\
Total leukocyte count, $10^{3} / \mathrm{mm}^{3}, \mathrm{~T} 1$ & $15.92(15.37)$ & $15.35(9.27)$ & 0.90 \\
Total leukocyte count, $10^{3} / \mathrm{mm}^{3}, \mathrm{~T} 2$ & $14.08(9.05)$ & $16.76(8.53)$ & 0.42 \\
Lymphocyte count, $10^{3} / \mathrm{mm}^{3}, \mathrm{~T} 1$ & $0.85(0.53)$ & $1.00(0.93)$ & 0.60 \\
Lymphocyte count, $10^{3} / \mathrm{mm}^{3}, \mathrm{~T} 2$ & $0.90(0.53)$ & $0.72(0.45)$ & 0.34 \\
C-reactive protein, $\mathrm{mg} / \mathrm{L}, \mathrm{T} 1$ & $245.2(127.1)$ & $217.0(156.0)$ & 0.59 \\
C-reactive protein, $\mathrm{mg} / \mathrm{L}, \mathrm{T} 2$ & $224.2(110.2)$ & $248.1(127.1)$ & 0.59 \\
Fibrinogen, $\mathrm{mg} / \mathrm{L}, \mathrm{T} 1$ & $5.34(2.30)$ & $4.60(1.10)$ & 0.27 \\
Fibrinogen, $\mathrm{mg} / \mathrm{L}, \mathrm{T} 2$ & $5.98(2.28)$ & $5.81(2.06)$ & 0.85 \\
\hline
\end{tabular}

Abbreviations: APACHE II Acute Physiology and Chronic Health Evaluation II, ICU intensive care unit, $\mathrm{paO}_{2} / \mathrm{FiO}_{2}$ partial pressure of oxygen/fraction of inspired oxygen, SOFA Sequential Organ Function Assessment, $\mathrm{SvCO}_{2}$ central venous oxygen saturation

Clinical characteristics of the patients divided in responders and non-responders at the two time points. Data are presented as mean (standard deviation) or frequency (percentage)

\section{Gene expression analysis over time}

We compared gene expression profiles between T2 and T1 in the R and NR groups independently. This analysis generated two lists of DEGs with 1979 DEGs (865 upregulated and 1114 downregulated genes) in Rs (Additional file 4: Table S2) and 1914 DEGs (711 upregulated and 1203 downregulated genes) in NRs (Additional file 5: Table S3). The two lists of DEGs were filtered at a padj $<10^{-3}$, yielding 1146 and 1120 genes in Rs and NRs, respectively, and were used as input for an ORA. In Rs, 14 clusters of significantly over-represented GO terms were identified with padj $<0.05$ (Table 2; Additional file 6: Table S4). In NRs, the same analysis identified 15 clusters of over-represented GO terms (Table 3; Additional file 7: Table S5). A comparison between the GO networks identified in Rs and NRs was performed in Cytoscape, highlighting GO terms specifically enriched exclusively in Rs or NRs or enriched in both groups (Figs. 2 and 3). In Rs, we identified three robust $\mathrm{GO}$ clusters with highly interconnected GO terms (positive regulation of

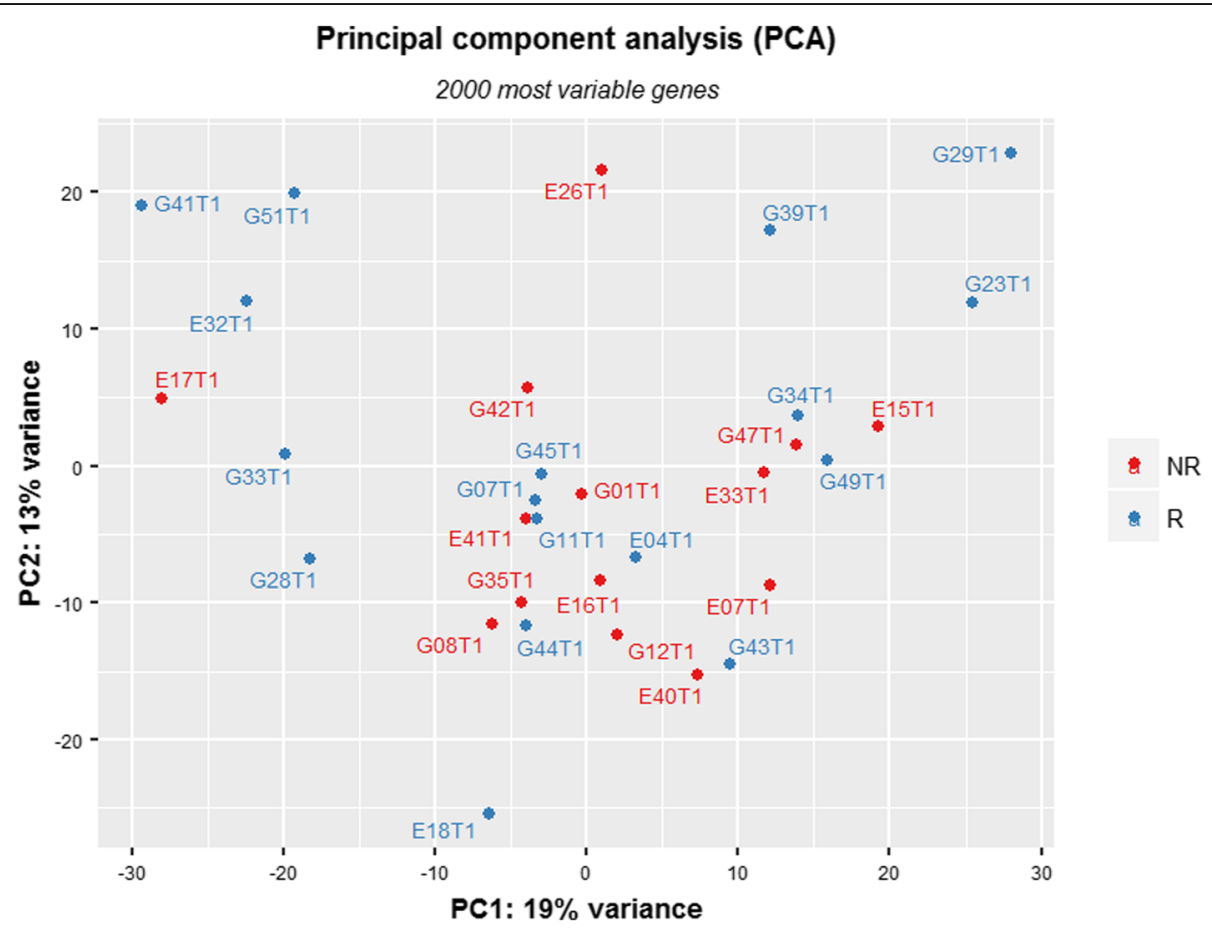

Fig. 1 Principal component analysis of gene expression data in responders (Rs) and non-responders (NRs) at time point 1 (T1). The figure presents the two major principal components using the set of 2000 most variable genes (in expression) across all samples. Red describes NR patients whereas blue describes R patients. R and NR patients do not exhibit separation on the PC1 and PC2 
Table 2 Gene Ontology analysis in responder patients

\begin{tabular}{|c|c|c|c|c|}
\hline GO groups & Group-adjusted $P$ value & Leading GO ID & Leading GO term & Leading GO genes \\
\hline Group 00 & $6.37 \mathrm{E}-5$ & GO:0001820 & Serotonin secretion & 6 \\
\hline Group 01 & $3.99 \mathrm{E}-5$ & GO:0006650 & Glycerophospholipid metabolic process & 44 \\
\hline Group 02 & $4.59 \mathrm{E}-5$ & GO:0042981 & Regulation of apoptotic process & 124 \\
\hline Group 03 & 7.67E-5 & GO:0043281 & $\begin{array}{l}\text { Regulation of cysteine-type endopeptidase } \\
\text { activity involved in apoptotic process }\end{array}$ & 29 \\
\hline Group 04 & $8.10 \mathrm{E}-5$ & GO:0002237 & Response to molecule of bacterial origin & 40 \\
\hline Group 05 & $5.76 \mathrm{E}-5$ & GO:0007169 & $\begin{array}{l}\text { Transmembrane receptor protein tyrosine kinase } \\
\text { signaling pathway }\end{array}$ & 70 \\
\hline Group 06 & $6.17 \mathrm{E}-5$ & GO:0038083 & Peptidyl-tyrosine autophosphorylation & 14 \\
\hline Group 07 & $6.05 E-5$ & GO:0042089 & Cytokine biosynthetic process & 20 \\
\hline Group 08, Group13 & 1.12E-7, 1.25E-16 & GO:0002696 & Positive regulation of leukocyte activation & 48 \\
\hline Group 09 & $8.82 \mathrm{E}-20$ & GO:0042119 & Neutrophil activation & 92 \\
\hline Group10 & $6.79 \mathrm{E}-5$ & GO:0050663 & Cytokine secretion & 30 \\
\hline Group11 & $3.85 \mathrm{E}-12$ & GO:0002253 & Activation of immune response & 81 \\
\hline Group12 & $1.44 \mathrm{E}-9$ & GO:0009967 & Positive regulation of signal transduction & 149 \\
\hline Group13 & $1.25 \mathrm{E}-16$ & GO:0030217 & T-cell differentiation & 43 \\
\hline
\end{tabular}

Gene Ontology (GO) analysis was performed on the most significant 1146 differentially expressed genes in responders between time point 1 (T1) and T2. Each line reports a significantly enriched GO group with its adjusted $P$ value. The name of the most significant GO term in the GO group is reported together with the number of genes in the GO term

leukocyte activation, activation of immune response, and T-cell differentiation) (Fig. 2) that were not identified in NRs (Table 2), suggesting that the adaptive immune response is activated mostly in Rs at T2.

In NRs, we could not identify any GO cluster with highly interconnected GO terms with the exception of clusters of signal transduction and regulated exocytosis, which share many GO terms with Rs (Fig. 3). Among the GO clusters specifically enriched only in NRs, we highlighted the "Response to lipopolysaccharide" GO cluster, including CCL5, CXCL1, CXCL5, IRAK1, and NLRP3 genes that were exclusively downregulated in NRs. Analysis of the 752 DEGs common to Rs and NRs identified eight clusters of significantly enriched GO

Table 3 Gene Ontology analysis in non-responder patients

\begin{tabular}{|c|c|c|c|c|}
\hline GO groups & Group-adjusted $P$ value & Leading GO ID & Leading GO term & Leading GO genes \\
\hline Group 00 & $1.83 \mathrm{E}-5$ & GO:0019221 & Cytokine-mediated signaling pathway & 69 \\
\hline Group 01 & $1.14 \mathrm{E}-4$ & GO:0006633 & Fatty acid biosynthetic process & 20 \\
\hline Group 02 & $4.52 \mathrm{E}-5$ & GO:0030100 & Regulation of endocytosis & 28 \\
\hline Group 03 & $1.32 \mathrm{E}-6$ & GO:0030168 & Platelet activation & 28 \\
\hline Group 04 & $1.08 \mathrm{E}-4$ & GO:0033032 & Regulation of myeloid cell apoptotic process & 9 \\
\hline Group 05 & 7.87E-6 & GO:0002576 & Platelet degranulation & 23 \\
\hline Group 06 & $8.84 \mathrm{E}-6$ & GO:0010506 & Regulation of autophagy & 39 \\
\hline Group 07 & $7.56 \mathrm{E}-5$ & GO:0002218 & Activation of innate immune response & 34 \\
\hline Group 08 & $1.18 \mathrm{E}-5$ & GO:0071222 & Cellular response to lipopolysaccharide & 29 \\
\hline Group 09 & 4.65E-5 & GO:0030334 & Regulation of cell migration & 69 \\
\hline Group10 & $5.26 \mathrm{E}-5$ & GO:0042981 & Regulation of apoptotic process & 122 \\
\hline Group11 & $1.29 \mathrm{E}-6$ & GO:0043281 & $\begin{array}{l}\text { Regulation of cysteine-type endopeptidase } \\
\text { activity involved in apoptotic process }\end{array}$ & 33 \\
\hline Group12 & $1.78 \mathrm{E}-5$ & GO:1904951 & Positive regulation of establishment of protein localization & 56 \\
\hline Group13 & $5.59 \mathrm{E}-25$ & GO:0045055 & Regulated exocytosis & 122 \\
\hline Group14 & $3.21 \mathrm{E}-10$ & GO:1902531 & Regulation of intracellular signal transduction & 152 \\
\hline
\end{tabular}

Gene Ontology (GO) analysis was performed on the most significant 1120 differentially expressed genes in non-responders between time point 1 (T1) and T2. Each line reports a significantly enriched GO group with its adjusted $P$ value. The name of the most significant GO term in the GO group is reported together with the number of genes in the GO term 


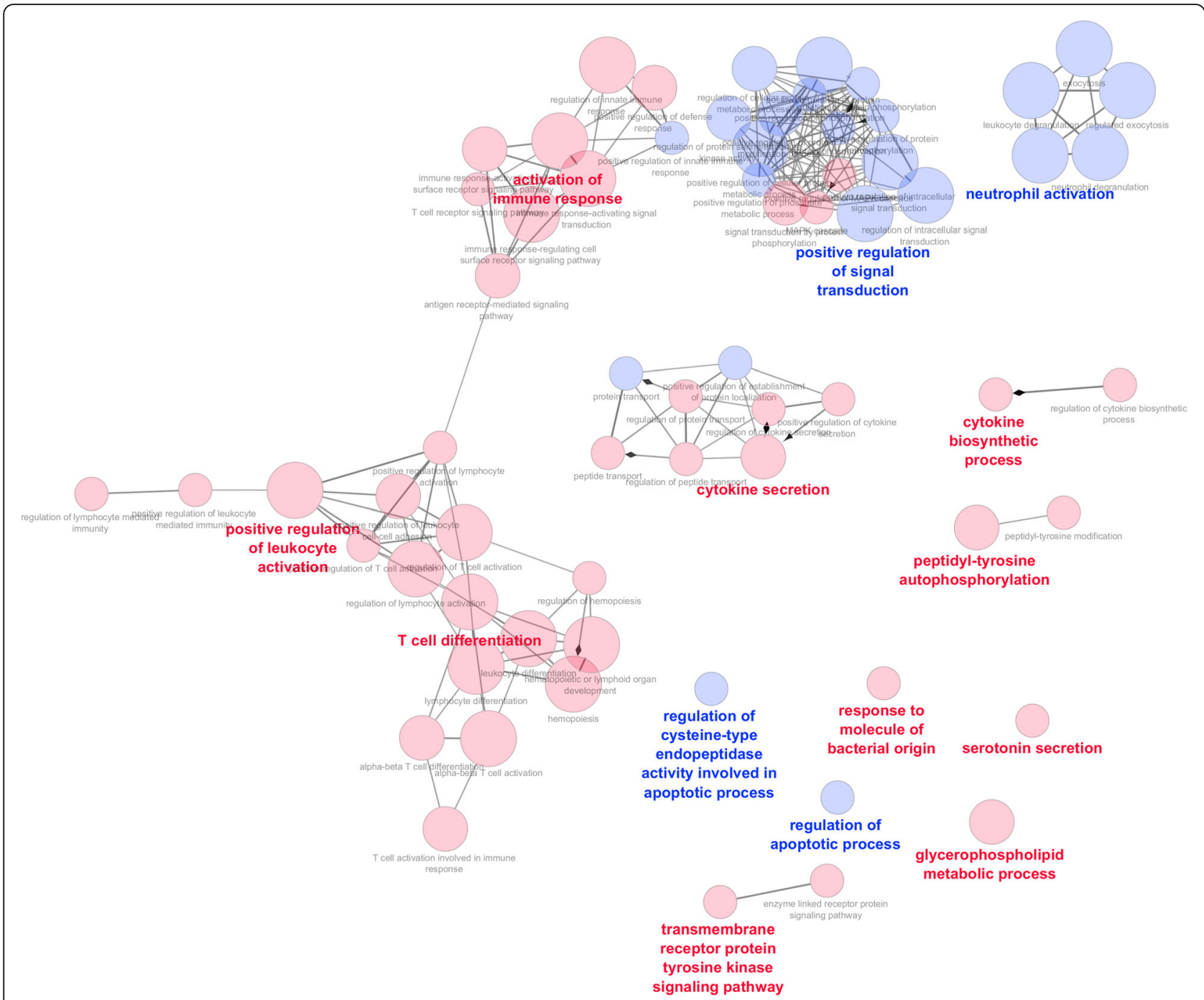

Fig. 2 Network of enriched Gene Ontology (GO) term clusters in responder (R) patients. In this plot, each circle corresponds to an enriched GO term. GO terms enriched exclusively in R patients are noted in red, whereas those common to non-responder (NR) patients are noted in blue. The most significant enriched GO terms (leading GO terms) in each GO cluster are noted in bold

terms (Table 4) that are related to modulation of genes involved in the innate immune response and neutrophil function.

\section{Gene expression trend analysis}

We identified 125 genes $(P<0.01$, Mann-Whitney) with significantly different trends between Rs and NRs in the transition from T1 to T2 (Fig. 4; Additional file 8: Table S6). Thirteen genes (CD247, FCER1A, ICOS, LCK, SH2D1A, CLC, IRAK3, VSIG4, GPR171, GPR18, HIST1H3A, ZN F784, and TIGIT) among 125 are involved in T-cell functions and support the results highlighted in the GO analysis that Rs modulate biological processes of the adaptive immune response.

We observed that, among the 125 genes, genes involved in granulocyte functions (IL5RA, FCER1A, CCR3, and
PTGDR2) and genes involved in natural killer (NK) cell activity (GZMM, GZMH, and KLRG1) were significantly upregulated at T2 exclusively in Rs. We also identified PCSK9 and SORT1 genes, which are involved in the lipid pathway. PCSK9 is significantly downregulated in Rs at T2. In contrast, SORT1 is downregulated at T2 in both groups, but different fold changes are noted for each group. We also observed genes with a role in the maintenance of vascular tone and endothelial function, such as CPA3 (upregulated at T2 in Rs), ARG1 (downregulated at $\mathrm{T} 2$ in Rs), and AVPR1A (downregulated at T2 in NRs).

\section{Validation in an external dataset}

Patients of the validation (GSE57065) and of the present study had similar clinical characteristics except for the SOFA score at $\mathrm{T} 1$ in Rs, which was higher in the present 


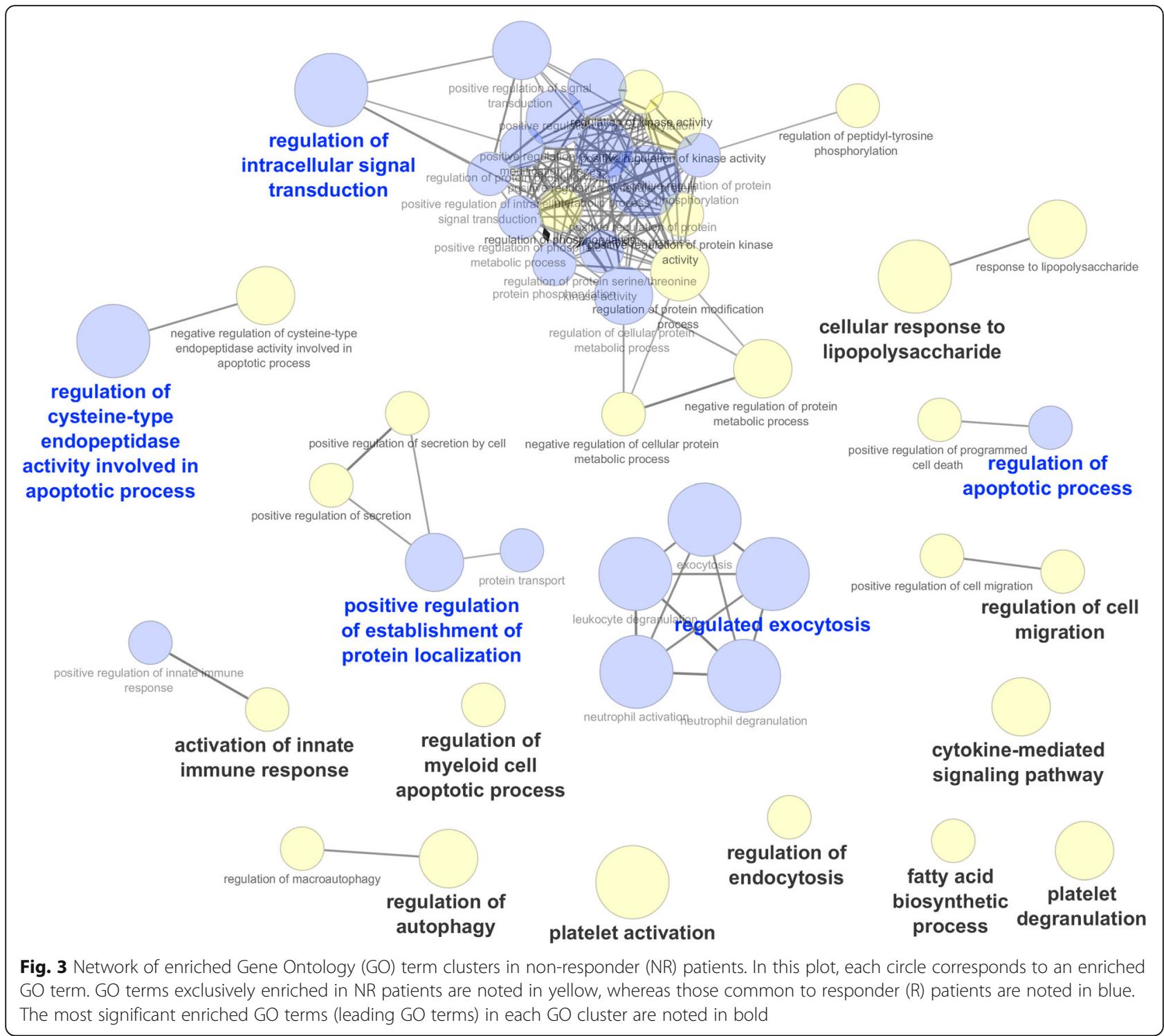

cohort compared with the validation one (11.58 \pm 3 versus $9.29 \pm 2.49, P=0.026)$. Gene expression comparison between $\mathrm{H} 0$ and $\mathrm{H} 48$ in the validation cohort generated two lists of 590 DEGs (327 upregulated and 263 downregulated) in Rs and 996 DEGs (819 upregulated and 178 downregulated) in NRs. To assess concordance of gene ID and direction of regulation, we compared these two lists with the DEG lists obtained for the R and NR groups in our study. We identified 234 DEGs modulated in Rs and 245 DEGs modulated in NRs in both datasets (padj < $0.05[\mathrm{BH}]$ and $\log \mathrm{FC}>|0.5|$ ). The concordance (upregulation/downregulation) was high (231 out of 234 for Rs and 242 out of 245 for NRs).

\section{Discussion}

We explored the transcriptome profile associated with early response to hemodynamic supportive therapy using whole blood of patients with septic shock to identify gene expression profiles that distinguish Rs from NRs over the first $48 \mathrm{~h}$ from ICU admission. This time window is crucial for critically ill patients given that those who exhibit improved organ function exhibit a better outcome [5, 19-21]. To quantify gene expression, we used RNA-Seq, which, compared with microarray, offers increased dynamic range of signal and reproducibility [22]. Moreover, RNA-Seq can interrogate the whole transcriptome and not only those genes for which the probes are designed on the microarray.

The comparison of the transcriptomic profile at ICU admission (T1) between Rs and NRs did not reveal any statistically significant difference. Previous studies with larger cohorts successfully identified a relation between gene expression profiles in leukocytes or whole blood cells at ICU admission and survival in sepsis and septic 
Table 4 Gene Ontology analysis on common differentially expressed genes between responder and non-responder patients

\begin{tabular}{|c|c|c|c|c|c|c|}
\hline GO groups & Group-adjusted $P$ value & GO ID & GO term & Number of genes & Leading GO term & $\begin{array}{l}\text { Term-adjusted } \\
P \text { value }\end{array}$ \\
\hline Group 00 & $6.44 \mathrm{E}-05$ & GO:0050663 & Cytokine secretion & 22 & $x$ & 1.11E-02 \\
\hline Group 01 & 7.17E-05 & GO:0051248 & $\begin{array}{l}\text { Negative regulation of protein metabolic } \\
\text { process }\end{array}$ & 73 & $x$ & 1.64E-02 \\
\hline Group 02 & $3.01 \mathrm{E}-06$ & GO:0002576 & Platelet degranulation & 19 & $x$ & 4.18E-04 \\
\hline Group 03 & $6.65 \mathrm{E}-05$ & GO:0030168 & Platelet activation & 18 & $x$ & 4.55E-02 \\
\hline Group 04 & 7.64E-05 & GO:0002237 & Response to molecule of bacterial origin & 30 & & 2.62E-02 \\
\hline Group 04 & 7.64E-05 & GO:0071219 & $\begin{array}{l}\text { Cellular response to molecule of bacterial } \\
\text { origin }\end{array}$ & 23 & $x$ & $5.42 \mathrm{E}-04$ \\
\hline Group 05 & 5.59E-08 & GO:0009967 & Positive regulation of signal transduction & 95 & $x$ & 4.14E-04 \\
\hline Group 05 & 5.59E-08 & GO:1902531 & $\begin{array}{l}\text { Regulation of intracellular signal } \\
\text { transduction }\end{array}$ & 107 & & 2.07E-03 \\
\hline Group 06 & 1.39E-25 & GO:0006887 & Exocytosis & 106 & & 7.79E-24 \\
\hline Group 06 & 1.39E-25 & GO:0042119 & Neutrophil activation & 78 & & $7.11 \mathrm{E}-23$ \\
\hline Group 06 & $1.39 \mathrm{E}-25$ & GO:0045055 & Regulated exocytosis & 102 & $x$ & $5.45 \mathrm{E}-26$ \\
\hline Group 06 & 1.39E-25 & GO:0043299 & Leukocyte degranulation & 81 & & $1.03 \mathrm{E}-22$ \\
\hline Group 06 & $1.39 \mathrm{E}-25$ & GO:0043312 & Neutrophil degranulation & 77 & & $1.32 \mathrm{E}-22$ \\
\hline Group 07 & 1.64E-06 & GO:0002253 & Activation of immune response & 54 & & $3.68 \mathrm{E}-04$ \\
\hline Group 07 & $1.64 \mathrm{E}-06$ & GO:0031349 & Positive regulation of defense response & 37 & & 2.91E-03 \\
\hline Group 07 & $1.64 \mathrm{E}-06$ & GO:0002757 & $\begin{array}{l}\text { Immune response-activating signal } \\
\text { transduction }\end{array}$ & 47 & & 5.24E-03 \\
\hline Group 07 & 1.64E-06 & GO:0045088 & Regulation of innate immune response & 39 & $x$ & 8.43E-05 \\
\hline Group 07 & 1.64E-06 & GO:0045089 & $\begin{array}{l}\text { Positive regulation of innate immune } \\
\text { response }\end{array}$ & 31 & & 3.38E-03 \\
\hline Group 07 & $1.64 \mathrm{E}-06$ & GO:0002218 & Activation of innate immune response & 28 & & 4.86E-03 \\
\hline
\end{tabular}

Gene Ontology (GO) analysis was performed on 752 differentially expressed genes identified between time point 1 (T1) and T2 in both responders and nonresponders. Each line reports a significantly enriched $\mathrm{GO}$ term with its adjusted $P$ value, the number of genes included and the GO group to which they belong

shock [23, 24]. Our negative result could be explained by the fact that our cohort is composed exclusively of septic shock patients highly homogeneous for disease severity, given that all patients exhibit high SOFA scores and are treated with vasopressors. Gene expression in the two groups likely reflects the homogeneity of the clinical phenotype. This result is consistent with that observed in a recent metabolomics study performed in a subgroup of these patients with identical metabolomic profiles at ICU admission [13].

Gene expression analysis over time was performed separately in Rs and NRs with a paired analysis leveraging the correlation between $\mathrm{T} 1$ and $\mathrm{T} 2$ and improving statistical power. The upregulation of neutrophil bacterial killing genes (CTSG, ELANE, DEFA4, and AZU1) and Toll-like receptor (TLR) genes involved in recognition of ssRNA (TLR7) and anti-inflammatory functions (TLR10) was detected at T2 in both Rs and NRs. In contrast, the following genes related to acute inflammation were downregulated: C-type lectin receptors (CLEC6A, CLEC4D, and CLEC5A), interferon receptors (IFNAR1 and IFNGR1), alarmins (S100A8 and S100A12), and metalloproteases involved in extracellular matrix remodeling (MMP8 and MMP9).

Interestingly, a comparison of the biological networks between Rs and NRs highlighted that R patients specifically modulate genes involved in T-cell activation but that NR patients do not, as observed in the trend analysis. In detail, ICOS and LCK, two important co-stimulatory molecules of T-cell activation [25, 26], and CD247, the major signal transduction element in the T-cell antigen receptor complex, were upregulated in Rs at T2. Modulation of these genes has been observed in relation to survival in previous articles [23, 27]. VSIG4, a strong inhibitory molecule of T-cell activation [28], was downregulated in Rs and upregulated in NRs at T2. The activation of adaptive immunity genes in Rs could suggest improved ability to eradicate infections [29] and reduce inflammation-induced organ injury. Consistent with this consideration, some genes with a relevant function in bacterial killing (CCL5, CXCL1, CXCL5, IRAK1, and NLRP3) were exclusively downregulated in NRs at T2.

The analysis of gene expression trends identified 125 genes differentiating the two groups of patients. However, 


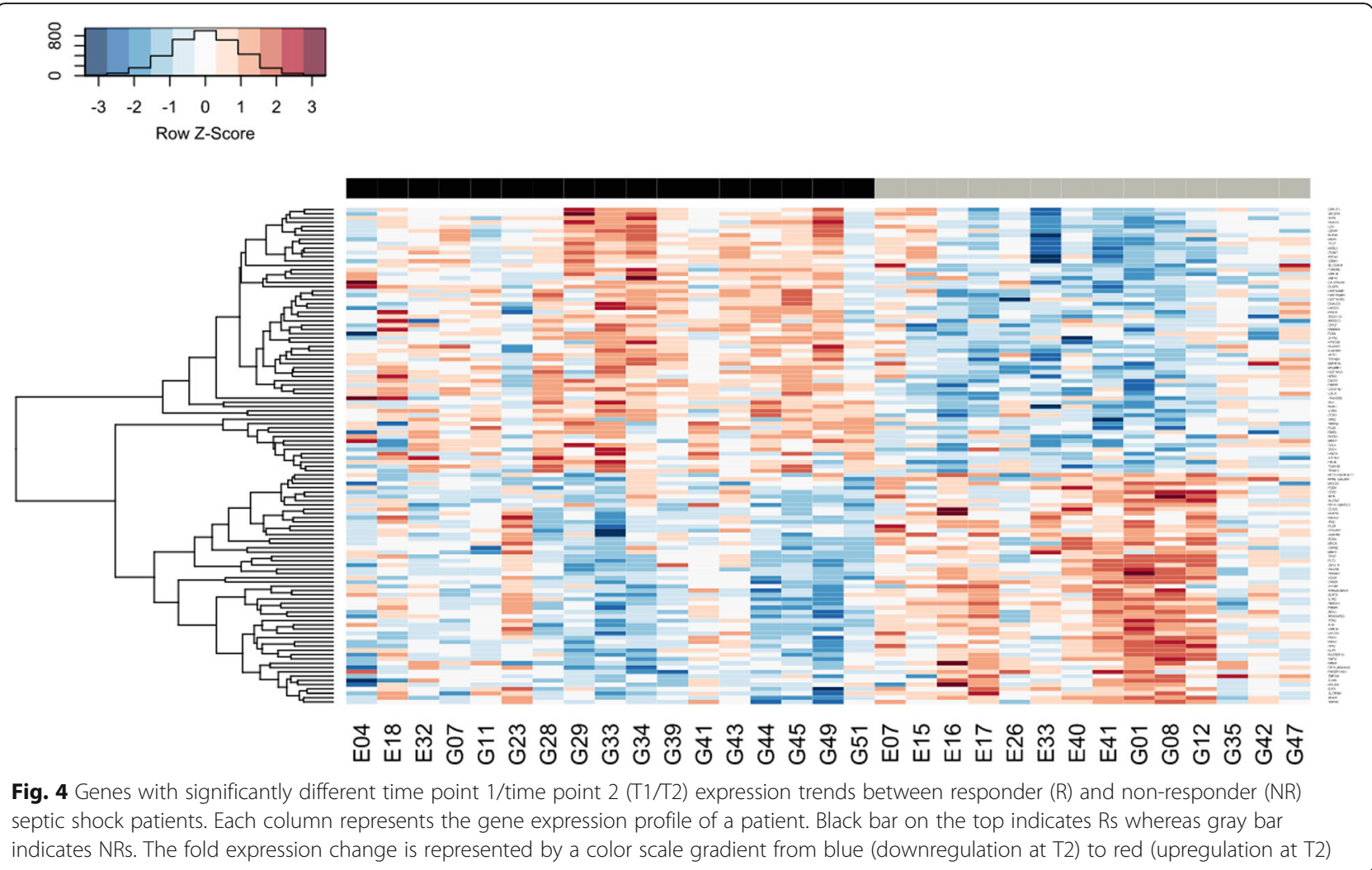

a certain degree of heterogeneity of gene expression changes can be appreciated in each group (Fig. 4) that can likely be explained by the underlying causes of the shock condition and patient-specific factors [30, 31].

Among these genes, serin protease genes (GZMM, GZMH, and KLRG1) involved in NK cell activity and cell-mediated immune response [32] and genes expressed mainly in granulocytes (CLC, CCR3, PTGDR2, FCER1A, and IL5RA) were exclusively upregulated at T2 in R patients. Recent studies have reported the association between granulocytes and sepsis [33]. CLC (Charcot-Leyden crystal galectin) encodes a lysophospholipase that regulates the immune response through the recognition of cell surface glycans. CCR3, PTGDR2 (alias CRTH2), and FCER1A are associated with allergic processes and have been described in sepsis [34] and bacterial meningitis [35]. IL-5RA is the receptor of IL-5, which has been proposed as a viable therapy for sepsis and is required for attraction and activation of eosinophils by Th2 cells [36]. Among the genes that differentiate $R$ and NR patients, IL-1R2 was exclusively downregulated in R. It encodes interleukin 1 receptor type 2 , which acts as a decoy receptor for IL-1A, inhibiting its pro-inflammatory activity [37]. Recently, increased IL-1R2 expression has been noted in septic patients compared with controls [38], and IL-1R2 has been proposed as a new biomarker for sepsis diagnosis.
Trend analysis revealed that PCSK9 and SORT1, two genes involved in lipid clearance, are downregulated in Rs. PCSK9 inhibits the clearance of endogenous cholesterol lipids from plasma by decreasing the number of LDL receptors that are involved in the removal of bacterial lipids from the blood during infections $[39,40]$. SORT1 is a critical regulator of PCSK9 activity, promoting its secretion from primary hepatocytes [41]. In Rs, its modulation combined with the downregulation of PCSK9 could promote increased clearance of endogenous lipids and bacteria. Indeed, in our patient cohort, lipidome alterations were found to play an important role in individual patient response to infection [13].

Hypotension [42] and impairment of endothelial function $[43,44]$ are key pathophysiological features of septic shock. Exclusively in R patients, CPA3 encoding a carboxypeptidase that degrades toxic peptides in vivo was upregulated at T2. ARG1 and AVPR1A were downregulated in Rs and NRs, respectively. ARG1 encodes arginase that affects nitric oxide generation [45], impacting the regulation of vascular tone and maintenance of endothelial function [46]. The vasopressin receptor AVPR1A is also expressed in platelets [47] and is responsible for the vasoconstrictor activity of vasopressin [47, 48]. Decreased expression of AVPR1A receptors and reduced levels of vasopressin have been described in experimental models of sepsis and human sepsis, respectively [47, 49]. 
Because the relatively small sample size of our study may be considered a limitation, we validated the DEGs identified and highlighted in the discussion, using the external dataset GSE57065. We validated genes involved in bacterial killing and anti-inflammatory functions (DEFA4, AZU1, ELANE, CTSG, and TLR10), C-type lectin receptor genes (CLEC4D and CLEC5A), the interferon receptor gene IFNAR1, the metalloprotease gene MMP8, genes involved in lipid clearance (PCSK9 and SORT1), and the alarmin gene S100A8.

Moreover, to further confirm our results, we focused on the GAinS study [23] that investigated gene expression in 265 septic patients at ICU admission and identified two sepsis signatures: SRS1 associated with increased mortality and SRS2. Interestingly, genes involved in T-cell function (CD247, SH2D1A, ICOS, TIGIT, LCK, and CLC), which were associated with improvement of organ function in $\mathrm{R}$ patients in our cohort, were also associated with survival in the GAinS study, suggesting that their early modulation is critical for a favorable outcome. Furthermore, genes involved in granulocyte function (FCER1A and CCR3), NK cell activity (GZMM, GZMH, and KLRG1), lipid pathways (PCSK9 and SORT1), and endothelial function (ARG1 and CPA3) exhibited the same direction of modulation in survivors and the $\mathrm{R}$ group.

We chose to categorize our cohort according to changes in organ function and not mortality. The association between ICU mortality and whole blood transcriptomics has been previously described $[9,23]$, and major changes have been observed within the first $48 \mathrm{~h}$ of shock development [8]. However, the reason for this association remains largely unexplored. We hypothesized that this could be due to different trajectories of organ (dys)function in response to standard therapy in the first days of ICU stay, which is a key determinant of outcome $[5,21,50]$. However, the mechanisms remain incompletely characterized. In a recent article in Critical Care, treatment effects of different interventions on delta-SOFA are "reliably and consistently associated with mortality", and delta-SOFA has been recommended as an endpoint in randomized controlled trials [51]. Finally, as the prevalence of ICU mortality decreases, methodological issues regarding statistical power are relevant. Hence, studying "intermediate" phenotypes that are more common than mortality and yet closely related is justified [52].

\section{Conclusions}

This study highlights a specific transcriptome profile associated with a positive response to supportive therapy. In short, this profile is characterized by the activation in $\mathrm{R} \mathrm{pa-}$ tients of genes involved in T cell-mediated immunity and of genes related to granulocyte and NK cell function and by the modulation of genes involved in pathogen lipid clearance, which contribute to a more efficient infection eradication and inflammation modulation.

\section{Additional files}

\begin{abstract}
Additional file 1: Supplementary methods. (DOCX $15 \mathrm{~kb}$ )
Additional file 2: Table S1. Metrics and basic statistics of sequencing data. This table reports the number of reads processed along the data analysis workflow. In particular, it presented the number of sequenced reads (raw reads), the number of quality filters passing reads (trimmed reads), and the number of reads that map to exons (column E). Columns $\mathrm{F}$ to I report the percentage of reads mapping to rRNA and the rate of mapping along genic or intergenic regions. (XLSX $16 \mathrm{~kb}$ )
\end{abstract}

Additional file 3: Table S7. Globin depletion (Mann-Whitney test). This table summarizes the percentage of reads mapping unambiguously to the reference genome (GRCh38) regarding all genes encoding for globins. For each gene, the following information is reported: mean percentage in responders (Rs) and non-responders (NRs), standard deviation in Rs and NRs, and $P$ value (Mann-Whitney test) of the comparison test between Rs and NRs. (XLSX 17 kb)

Additional file 4: Table S2. Differentially expressed gene (DEG) T2vsT1 in responder (R) patients. This table describes the results of the differential expression analysis between T1 and T2 in Rs. In total, 1979 DEGs were identified with a padj $<0.01$. For each gene, the following information is reported: Ensembl Gene Name, the mean level of expression in all samples (baseMean), log2FoldChange comparing T2 versus T1, level of statistical significance (padj), genomic coordinates (chromosome_name, start_position, end_position), description of gene function (description), official gene symbol (external_gene_name), type of gene (gene_biotype), and the normalized gene count values in each biological sample. Abbreviations: 11 time point 1, T2 time point 2. (XLSX $2787 \mathrm{~kb}$ )

Additional file 5: Table S3. Differentially expressed gene (DEG) T2vsT1 in non-responder (NR) patients. This table describes the results of the differential expression analysis between T1 and T2 in NRs. In total, 1914 DEGs were identified with a padj $<0.01$. For each gene, the following information is reported: Ensembl Gene Name, the mean level of expression in all samples (baseMean), log2FoldChange comparing T2 versus T1, level of statistical significance (padj), genomic coordinates (chromosome_name, start_position, end_position), description of gene function (description), official gene symbol (external_gene_name), type of gene (gene_biotype), and the normalized gene count values in each biological sample. Abbreviations: T1 time point 1, T2 time point 2. (XLSX $2409 \mathrm{~kb}$ )

Additional file 6: Table S4. Gene Ontology (GO) enriched clusters obtained from differentially expressed gene (DEG) list - full table in responder (R) patients. This table is an extended and more detailed version of Table 2. All of the 66 significantly enriched $\mathrm{GO}$ terms are described, and we specify whether the GO term is found exclusively in Rs or in both Rs and non-responders (NRs) (Patient condition). (XLSX 17 kb)

Additional file 7: Table S5. Gene Ontology (GO) enriched clusters obtained from differentially expressed gene (DEG) list - full table in nonresponder (NR) patients. This table is an extended and more detailed version of Table 3. All of the 48 significantly enriched $\mathrm{GO}$ terms are described, and we specify whether the $\mathrm{GO}$ term is found exclusively in NRs or in both responders (Rs) and NRs (Patient condition). (XLSX 16 kb)

Additional file 8: Table S6. Trend analysis (Mann-Whitney test) - full table. This table describes the 125 genes exhibiting different expression trends in responders (Rs) and non-responders (NRs) between time point 1 (T1) and T2. For each gene, the following information is reported: log2FoldChange comparing T2 versus T1 in R (log2FC.R) and NR (log2FC.NR), the level of statistical significance in $R$ and NR (padj.R, padj.NR), the level of significance of the Mann-Whitney test (pval.wilcox), the mean level of expression in R (basemean R) and NR (baseMean NR), genomic coordinates (chromosome, start, end), description of gene function (description), and type of gene (gene_biotype). (XLSX $33 \mathrm{~kb}$ )

\section{Abbreviations}

BH: Benjamini-Hochberg; DEG: Differentially expressed gene; FDR: False discovery rate; GEO: Gene expression omnibus; GO: Gene Ontology; H: Hour; ICU: Intensive care unit; NK: Natural killer; NR: Non-responder; ORA: Overrepresentation analysis; $\mathrm{R}$ : Responder; RNA-Seq: RNA sequencing;

SOFA: Sequential organ function assessment; SRS: Sepsis response signature; T1: Time point 1; T2: Time point 2; TLR: Toll-like receptor 


\section{Acknowledgments}

Not applicable.

\section{Funding}

The study was supported by "ShockOmics" grant 602706 of the European Union.

\section{Availability of data and materials}

The datasets generated and analyzed during the current study are available through the Gene Expression Omnibus Database (accession number GSE110487).

\section{Authors' contributions}

$C B, K B, B B P, M B$, and $D B$ contributed to the study concept and design. $D B$, $\mathrm{DAF}$, and $\mathrm{FT}$ performed the laboratory work. BBP, $\mathrm{KB}$, and $\mathrm{AH}$ were in charge of the clinical work. $M B$ and $D B$ performed the statistical and bioinformatic analysis. GM and MAC provided the validation dataset. $C B, D B, M B, B B P$, and $\mathrm{KB}$ contributed to drafting the manuscript. All authors approved the final version of the manuscript.

\section{Ethics approval and consent to participate}

The Geneva Regional Research Ethics Committee (Commission cantonale d'éthique de la recherche, or CCER) (Geneva, Switzerland) and the Universite Libre de Bruxelles (Brussels, Belgium) approved the clinical protocol. Informed consent was obtained from the participants or their representatives.

\section{Consent for publication}

Not applicable.

\section{Competing interests}

The authors declare that they have no competing interests.

\section{Publisher's Note}

Springer Nature remains neutral with regard to jurisdictional claims in published maps and institutional affiliations.

\section{Author details}

'Dipartimento di Scienze della Salute, Università degli Studi di Milano, Via Rudini 8, 20142 Milan, Italy. ${ }^{2}$ Fondazione Filarete, Viale Ortles 22/4, 20139 Milan, Italy. ${ }^{3}$ Department of Anaesthesia, Pharmacology and Intensive Care, Geneva University Hospitals, Rue Gabrielle-Perret-Gentil 4, Geneva 1205 Switzerland. ${ }^{4}$ Laboratoire Commun de Recherche HCL-bioMérieux, Hôpital Edouard Herriot, 376 Chemin de I'Orme, 6928 Marcy-l'Etoile Lyon, France. ${ }^{5}$ Hospices Civils de Lyon, Hôpital Edouard Herriot, Laboratoire d'Immunologie, 5 Place d'Arsonval, 69437 Lyon cedex 03, France. ${ }^{6}$ Department of Intensive Care, Hospital Erasme, Hospital, Université Libre de Bruxelles, Route de Lennik 808, Brussels 1070, Belgium.

Received: 28 March 2018 Accepted: 16 October 2018 Published online: 21 November 2018

\section{References}

1. Singer M, Deutschman CS, Seymour CW, Shankar-Hari M, Annane D, Bauer $M$, et al. The Third International Consensus Definitions for Sepsis and Septic Shock (Sepsis-3). JAMA. 2016:315:801.

2. Seymour CW, Liu VX, Iwashyna TJ, Brunkhorst FM, Rea TD, Scherag A, et al. Assessment of Clinical Criteria for Sepsis: For the Third International Consensus Definitions for Sepsis and Septic Shock (Sepsis-3). JAMA. 2016; 315:762-74

3. Andreis DT, Singer M. Catecholamines for inflammatory shock: a Jekyll-andHyde conundrum. Intensive Care Med. 2016;42:1387-97.

4. Neyra JA, Li X, Canepa-Escaro F, Adams-Huet B, Toto RD, Yee J, et al. Cumulative Fluid Balance and Mortality in Septic Patients With or Without Acute Kidney Injury and Chronic Kidney Disease. Crit Care Med. 2016:44:1891-900.

5. Sakr Y, Lobo SM, Moreno R, Gerlach H, Ranieri VM, Michalopoulos A, et al. Patterns and early evolution of organ failure in the intensive care unit and their relation to outcome. Crit Care. 2012;16:R222.

6. Angus DC, Barnato AE, Bell D, Bellomo R, Chong CR, Coats TJ, et al. A systematic review and meta-analysis of early goal-directed therapy for septic shock: the ARISE, ProCESS and ProMISe Investigators. Intensive Care Med. 2015:41:1549-60.

7. Vincent JL, Grimaldi D. Novel Interventions: What's New and the Future. Crit Care Clin. 2018:34:161-73.

8. Cazalis M-A, Lepape A, Venet F, Frager F, Mougin B, Vallin H, et al. Early and dynamic changes in gene expression in septic shock patients: a genomewide approach. Intensive Care Med Exp. 2014;2:20.

9. Parnell GP, Tang BM, Nalos M, Armstrong NJ, Huang SJ, Booth DR, et al. Identifying key regulatory genes in the whole blood of septic patients to monitor underlying immune dysfunctions. Shock. 2013;40:166-74.

10. Braga D, Barcella M, Lupoli S, Herpain A, Bollen Pinto B, Baselli G, et al. Differential gene expression in septic shock patients according to early supportive therapy response. J Crit Care. 2017:42:378.

11. Aletti F, Conti C, Ferrario M, Ribas V, Bollen Pinto B, Herpain A, et al. ShockOmics: multiscale approach to the identification of molecular biomarkers in acute heart failure induced by shock. Scand J Trauma Resusc Emerg Med. 2016;24:9.

12. Vincent J, Moreno R, Takala J, Willatts S, De Mendonça A, Bruining H, et al. The SOFA (Sepsis-related Organ Failure Assessment) score to describe organ dysfunction/failure. On behalf of the Working Group on Sepsis-Related Problems of the European Society of Intensive Care Medicine. Intensive Care Med. 1996;22:707-10.

13. Cambiaghi A, Pinto BB, Brunelli L, Falcetta F, Aletti F, Bendjelid K, et al. Characterization of a metabolomic profile associated with responsiveness to therapy in the acute phase of septic shock. Sci Rep. 2017;7:9748.

14. Dobin A, Davis CA, Schlesinger F, Drenkow J, Zaleski C, Jha S, et al. STAR: Ultrafast universal RNA-seq aligner. Bioinformatics. 2013;29:15-21.

15. Liao Y, Smyth GK, Shi W. FeatureCounts: An efficient general purpose program for assigning sequence reads to genomic features. Bioinformatics. 2014;30:923-30.

16. Love Ml, Huber W, Anders S. Moderated estimation of fold change and dispersion for RNA-seq data with DESeq2. Genome Biol. 2014;15:550.

17. Bindea G, Mlecnik B, Hackl H, Charoentong P, Tosolini M, Kirilovsky A, et al. ClueGO: A Cytoscape plug-in to decipher functionally grouped gene ontology and pathway annotation networks. Bioinformatics. 2009; 25:1091-3.

18. Barrett T, Wilhite SE, Ledoux P, Evangelista C, Kim IF, Tomashevsky M, et al. NCBI GEO: Archive for functional genomics data sets - Update. Nucleic Acids Res. 2013;41(Database issue):D991-5.

19. Ferreira FL. Serial Evaluation of the SOFA Score to Predict Outcome in Critically III Patients. JAMA. 2001;286:1754.

20. Russell JA, Singer J, Bernard GR, Wheeler A, Fulkerson W, Hudson L, et al. Changing pattern of organ dysfunction in early human sepsis is related to mortality. Crit Care Med. 2000;28:3405-11.

21. Levy MM, Macias WL, Vincent J-L, Russell JA, Silva E, Trzaskoma B, et al. Early changes in organ function predict eventual survival in severe sepsis. Crit Care Med. 2005:33:2194-201.

22. Marioni JC, Mason CE, Mane SM, Stephens M, Gilad Y. RNA-seq: An assessment of technical reproducibility and comparison with gene expression arrays. Genome Res. 2008;18:1509-17.

23. Davenport EE, Burnham KL, Radhakrishnan J, Humburg P, Hutton P, Mills TC, et al. Genomic landscape of the individual host response and outcomes in sepsis: A prospective cohort study. Lancet Respir Med. 2016:4:259-71.

24. Tsalik EL, Langley RJ, Dinwiddie DL, Miller NA, Yoo B, van Velkinburgh JC, et al. An integrated transcriptome and expressed variant analysis of sepsis survival and death. Genome Med. 2014;6:111.

25. Dong C, Juedes AE, Temann UA, Shresta S, Allison JP, Ruddle NH, et al. ICOS co-stimulatory receptor is essential for T-cell activation and function. Nature. 2001;409:97-101.

26. Lovatt M, Filby A, Parravicini V, Werlen G, Palmer E, Zamoyska R. Lck Regulates the Threshold of Activation in Primary T Cells, While both Lck and Fyn Contribute to the Magnitude of the Extracellular Signal-Related Kinase Response. Mol Cell Biol. 2006;26:8655-65.

27. Almansa R, Heredia-Rodriguez M, Gomez-Sanchez E, Andaluz-Ojeda D, Iglesias V, Rico L, et al. Transcriptomic correlates of organ failure extent in sepsis. J Inf Secur. 2015:70:445-56.

28. Vogt L, Schmitz N, Kurrer MO, Bauer M, Hinton HI, Behnke S, et al. VSIG4, a B7 family-related protein, is a negative regulator of $\mathrm{T}$ cell activation. J Clin Invest. 2006;116:2817-26.

29. Kasten KR, Tschöp J. Adediran SG, Hildeman DA Caldwell CC T cells are potent early mediators of the host response to sepsis. Shock. 2010;34:327-36. 
30. Sims CR, Nguyen TC, Mayeux PR. Could Biomarkers Direct Therapy for the Septic Patient? J Pharmacol Exp Ther. 2016;357:228-39.

31. Marshall JC, Reinhart K. Biomarkers of sepsis. Crit Care Med. 2009;37:2290-8.

32. Chowdhury D, Lieberman J. Death by a Thousand Cuts: Granzyme Pathways of Programmed Cell Death. Annu Rev Immunol. 2008;26:389-420.

33. Nierhaus A, Klatte S, Linssen J, Eismann NM, Wichmann D, Hedke J, et al. Revisiting the white blood cell count: immature granulocytes count as a diagnostic marker to discriminate between SIRS and sepsis - a prospective, observational study. BMC Immunol. 2013;14:8.

34. Venet F, Lepape A, Debard AL, Bienvenu J, Bohé J, Monneret G. The Th2 response as monitored by CRTH2 or CCR3 expression is severely decreased during septic shock. Clin Immunol. 2004;113:278-84.

35. Lill M, Köks S, Soomets U, Schalkwyk LC, Fernandes C, Lutsar I, et al. Peripheral blood RNA gene expression profiling in patients with bacterial meningitis. Front Neurosci. 2013:7:33.

36. Linch SN, Danielson ET, Kelly AM, Tamakawa RA, Lee JJ, Gold JA. Interleukin 5 is protective during sepsis in an eosinophil-independent manner. Am J Respir Crit Care Med. 2012;186:246-54

37. Garlanda C, Dinarello CA, Mantovani A. The Interleukin-1 Family: Back to the Future. Immunity. 2013;39:1003-18.

38. Lang $Y$, Jiang $Y$, Gao $M$, Wang $W$, Wang $N$, Wang $K$, et al. Interleukin-1 Receptor 2: A New Biomarker for Sepsis Diagnosis and Gram-Negative/ Gram-Positive Bacterial Differentiation. Shock. 2017;47:119-24.

39. Walley KR, Thain KR, Russell JA, Reilly MP, Meyer NJ, Ferguson JF, et al. PCSK9 is a critical regulator of the innate immune response and septic shock outcome. Sci Transl Med. 2014:6:258ra143.

40. Khademi F, Momtazi-borojeni AA, Reiner Ž, Banach M, Al A-RK, Sahebkar A. PCSK9 and infection: A potentially useful or dangerous association? J Cell Physiol. 2017;233:2920-7.

41. Gustafsen C, Kjolby M, Nyegaard M, Mattheisen M, Lundhede J, Buttenschøn $\mathrm{H}$, et al. The hypercholesterolemia-risk gene SORT1 facilitates PCSK9 secretion. Cell Metab. 2014;19:310-8.

42. Sharawy N. Vasoplegia in septic shock: Do we really fight the right enemy? J Crit Care. 2014;29:83-7.

43. Matsuda N, Hattori Y. Vascular biology in sepsis: pathophysiological and therapeutic significance of vascular dysfunction. J Smooth Muscle Res. 2007; 43:117-37.

44. Ait-Oufella H, Maury E, Lehoux S, Guidet B, Offenstadt G. The endothelium: Physiological functions and role in microcirculatory failure during severe sepsis. Intensive Care Med. 2010;36:1286-98.

45. Rath M, Müller I, Kropf P, Closs El, Munder M. Metabolism via arginase or nitric oxide synthase: Two competing arginine pathways in macrophages. Front Immunol. 2014;5:532.

46. Tousoulis D, Kampoli A-M, Tentolouris C, Papageorgiou N, Stefanadis C. The role of nitric oxide on endothelial function. Curr Vasc Pharmacol. 2012;10:4-18.

47. Russell JA, Walley KR. Vasopressin and its immune effects in septic shock. Innate Immun. 2010;2:446-60.

48. Maybauer MO, Maybauer DM, Enkhbaatar P, Traber DL. Physiology of the vasopressin receptors. Best Pract Res Clin Anaesthesiol. 2008;22:253-63.

49. Bucher M, Hobbhahn J, Taeger K, Kurtz A. Cytokine-mediated downregulation of vasopressin $\mathrm{V}(1 \mathrm{~A})$ receptors during acute endotoxemia in rats. Am J Physiol Regul Integr Comp Physiol. 2002;282:R979-84.

50. Moreno R, Vincent JL, Matos R, Mendonça A, Cantraine F, Thijs L, et al. The use of maximum SOFA score to quantify organ dysfunction/failure in intensive care. Results of a prospective, multicentre study. Intensive Care Med. 1999;25:686-96.

51. de Grooth H-J, Geenen IL, Girbes AR, Vincent J-L, Parienti J-J, Oudemans-van Straaten HM. SOFA and mortality endpoints in randomized controlled trials: a systematic review and meta-regression analysis. Crit Care. 2017;21:38.

52. Machado F, Sanches L, Azevedo L, Bruniatti M, Lourenço D, Noguti M, et al. Association between organ dysfunction and cytokine concentrations during the early phases of septic shock. Rev Bras Ter Intensiva. 2011;23:426-33.

Ready to submit your research? Choose BMC and benefit from:

- fast, convenient online submission

- thorough peer review by experienced researchers in your field

- rapid publication on acceptance

- support for research data, including large and complex data types

- gold Open Access which fosters wider collaboration and increased citations

- maximum visibility for your research: over $100 \mathrm{M}$ website views per year

At BMC, research is always in progress.

Learn more biomedcentral.com/submissions 